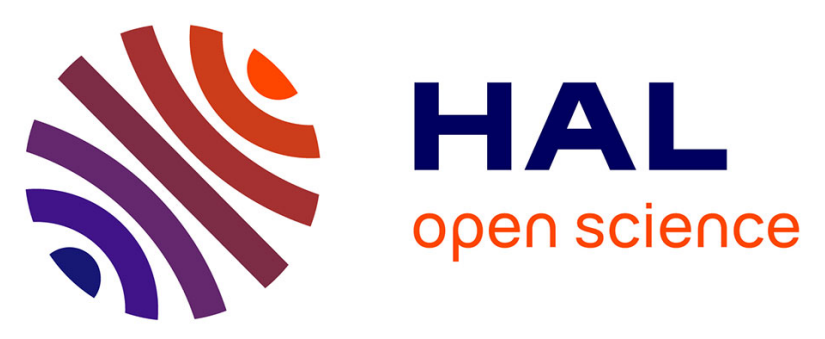

\title{
Iterative Multi-Stage Method for a Large Water Network Sectorization into DMAs under Multiple Design Objectives
}

Denis Gilbert, Edo Abraham, Idel Montalvo, Olivier Piller

\section{- To cite this version:}

Denis Gilbert, Edo Abraham, Idel Montalvo, Olivier Piller. Iterative Multi-Stage Method for a Large Water Network Sectorization into DMAs under Multiple Design Objectives. Journal of Water Resources Planning and Management, 2017, 143 (11), 04017067, 10 p. 10.1061/(ASCE)WR.19435452.0000835 . hal-01807869

\section{HAL Id: hal-01807869 \\ https://hal.science/hal-01807869}

Submitted on 5 Jun 2018

HAL is a multi-disciplinary open access archive for the deposit and dissemination of scientific research documents, whether they are published or not. The documents may come from teaching and research institutions in France or abroad, or from public or private research centers.
L'archive ouverte pluridisciplinaire HAL, est destinée au dépôt et à la diffusion de documents scientifiques de niveau recherche, publiés ou non, émanant des établissements d'enseignement et de recherche français ou étrangers, des laboratoires publics ou privés. 
Author-produced version of the article published in J. Water Res. Plan. \& Man., 2017, 143(11): 04017067. The original publication is available at http://ascelibrary.org/doi/full/10.1061/(ASCE)WR.19435452.0000835; doi: 10.1061/(ASCE)WR.1943-5452.0000835 


\title{
Iterative Multi-Stage Method for a Large Water Network Sectorization into \\ DMAs under Multiple Design Objectives
}

\section{Denis. Gilbert Ph.D. ${ }^{1}$, Edo Abraham Ph.D. ${ }^{2}$, Idel Montalvo Ph.D. ${ }^{3}$ and Olivier Piller Ph.D. ${ }^{4}$,}

${ }^{1}$ Research Engineer, Irstea, UR ETBX, Water Department, Bordeaux regional centre, Cestas F-33612, France, denis.gilbert@,irstea.fr

${ }^{2}$ Assistant Professor, Faculty of Civil Engineering and Geosciences, TU Delft, Stevinweg 1, 2628 CN Delft, the Netherlands, e.abraham@tudelft.nl

${ }^{3}$ Founder and Chief Engineer, IngeniousWare GmbH, Jollystraße 11, 76137 Karlsruhe, Germany, imontalvo@,ingeniousware.net

${ }^{4}$ Research scientist, Irstea, UR ETBX, Water Department, Bordeaux regional centre, Cestas F-33612, France, olivier.piller@,irstea.fr

\begin{abstract}
:
This paper considers the sectorization of a large water distribution network into DMAs and simultaneously optimizes rehabilitation of the network with new pipes, control valves, and storage tanks. Since the available water resources are much smaller in the dry season, both the design and operational settings are optimized to satisfy water demand, water quality and pressure constraints, and efficiency indices under stringent conditions. Because of the heterogeneity of the multiple decision variables and the complicated way they interact through the multiple objectives (some complimentary and some conflicting), it is not possible to fully automate the simultaneous sectorization, rehabilitation and operational optimization. Therefore, we employ a multi-stage approach where engineering judgment and network graph simplification and visualization tools are employed to find a good feasible solution that is used as a first guess for further optimization of sectors and operational settings, to achieve feasible solutions with better cost of implementation, demand similarity among DMAs and better pressure uniformity in operations. A multi-objective Agent Swarm Optimization (ASO) framework is used to iteratively change the sectors at the boundaries. For the final configuration, sequential linear programming is used to find optimal valve and pump settings.
\end{abstract}

Keywords: Water Distribution Network, Graph Partitioning, DMA, Sectorization, Rehabilitation Design, Agent Swarm Optimization, Pressure Management, Water Quality, Engineering Judgment 


\section{INTRODUCTION}

This manuscript presents our work on the optimal sectorization of the E-town network, which was part of the Battle of Water Networks DMAs (BWNDMA 2016), a special contest with 14 participants that was presented at WDSA 2016 in Cartagena, Colombia, the sixth one in a series of contests since 1985 (Giustolisi et al., 2015). Firstly, we describe the network and the problem objectives and constraints. Then, the methodology employed is explained, which consists of some engineering judgment and network analysis tools to simplify the problem, and the use of mathematical optimization and agent swarm optimization for design and operational decisions. We subsequently present our results and discuss lessons of general validity that have been learned from this exercise. Finally, we conclude with a summary.

The schematic shown in Figure 1 represents a large water network of the E-Town city in Colombia. As a result of a swelling tourist industry and overall economic growth, the city is no longer able to meet its increasing water demand. Having modelled the network and made an inventory of the forecasted demands, demand patterns, existing pump and tank characteristics, and the actual controls of valves, the municipality has determined that the current DMA configuration and operational and tactical management of the network do not allow it to meet demands in dry and rainy seasons (BWNDMA 2016). For example, as depicted in Figure 2, some of the tanks are empty and out of use because there are considerable differences in the pressure conditions of the city, and because the water use is not efficient. Therefore, the objective of this work was to sectorize the network into a new DMA configuration that allows the water utility to satisfy its customer needs while keeping a minimal number of DMAs (each with a similar number of users or demand), guarantying pressure uniformity across the municipality, meeting regulatory water quality specifications, and ensuring an efficient system operation during both the dry and wet seasons of the year. 


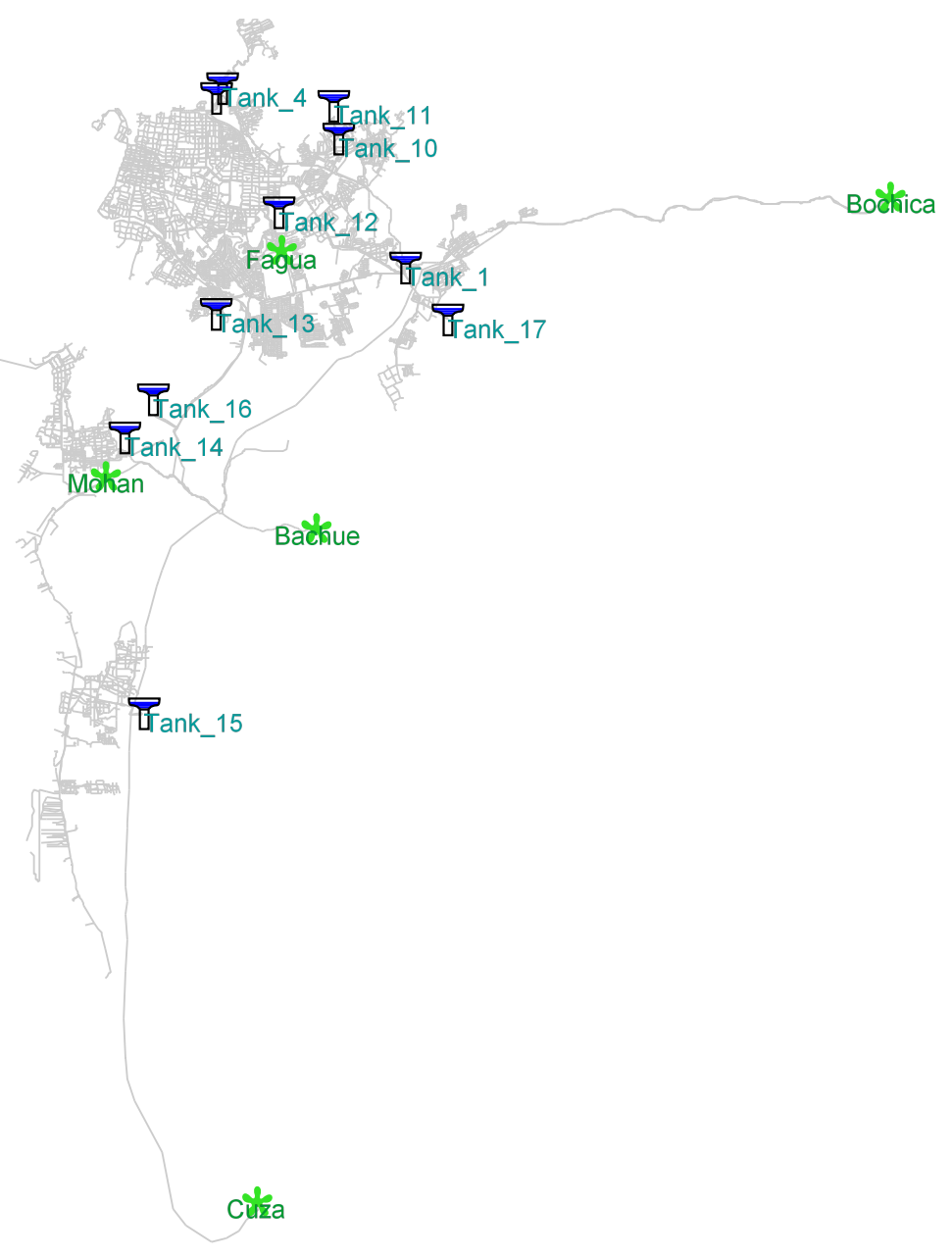

Figure 1: E-Town network Map with resources and used tanks.

The competition committee had specified a few decision variables and multiple cost functions for the design. The objectives that were evaluated to compute overall grades for the competitors were as follows (BWNDMA 2016):

i) To minimize the number of DMAs, subject to a minimum of 15 DMAs. The cost function given was $D M A_{\text {index }}=\# D M A s-15$

ii) To maximize demand similarity among DMAs. The cost function set was $D S=$ $\sqrt{\frac{1}{\# D M A S}} \sum_{i=1}^{\# D A S}\left(V_{i n, i}-V_{\text {out }, i}-V_{a v}\right)$, where $V_{i n, i}, V_{\text {out }, i}$ are the volume of water entering and leaving the ith DMA ands $V_{a v}$ volume consumed per DMA, averaged over all DMAs..

iii) To minimize solution implementation $\operatorname{cost} C C_{\text {net }}$. This consists of the cost of pipe replacements or installations (as a function of length and discrete set of available diameters), the cost of 
control valve installations (as a function of diameters), and the cost of new tanks installed (as a function of volume).

iv) To minimize the pressure Uniformity index $\left(P U_{\text {net }}\right)$ during the rainy and dry seasons as two separate objectives. These objectives specify that all demand nodes in the network should have similar pressure and as close as possible to the minimum set pressure constraint. Please see Equation (3) of this manuscript for details.

v) Minimize demand weighted water age $\left(W A_{\text {net }}\right)$ throughout the network. This minimizes the deviation of the water age from the local regulatory maximum age of 60 hours. Please see (BWNDMA 2016) for detailed expressions.

vi) Minimize the total number of operational changes $\left(\mathrm{OpCH}_{\text {net }}\right)$ in the valve settings (for pressure reducing valves (PRVs) and flow control valves (FCVs)) and opening and closing of isolation valves for boundary pipes from dry season to rainy season.

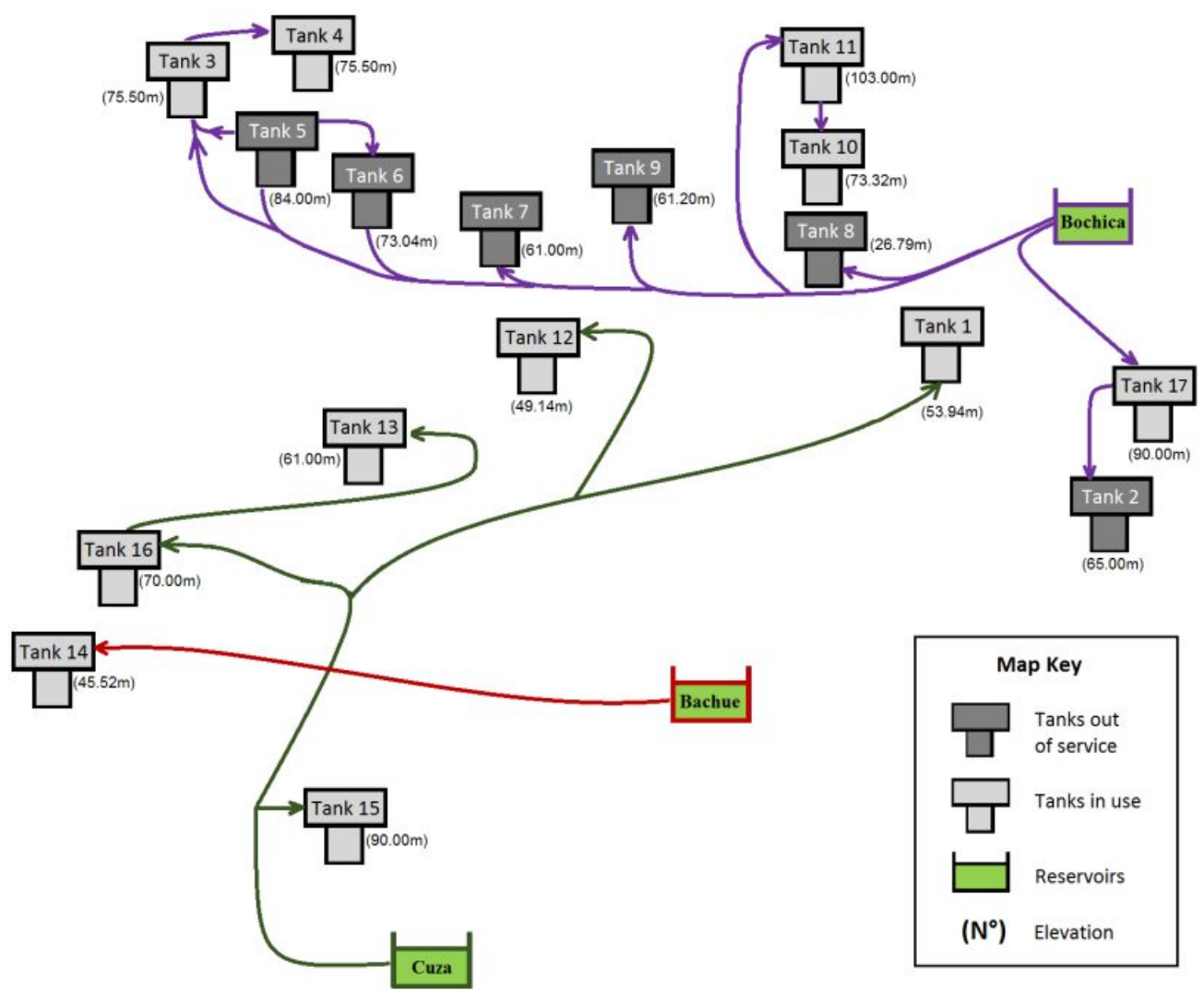

Figure 2: The original supply routes for the E-Town network (BWNDMA 2016) 
This design problem is a very difficult since there are multiple decision variables that are not easy to incorporate in one optimization problem and because the multiple objectives interact in complicated ways. For example, one of the design stage decision variables is the isolation valves to be closed (i.e. sectorization is done by closing some of the pipes on the boundary of DMAs). Since each DMA is specified to have only a maximum of two pressure controlled inlets, this will implicitly determine the number of PRVs and so the objective $C C_{\text {net }}$, in addition to $D M A_{\text {index }}$ and $D S$. The new tanks to be installed and their volume is not easy to incorporate in this same sectorization problem as it is not clear a priori, where additional storage is needed or what the volumes should be. Similarly, we have the discrete decisions of introducing additional new pipes. These design decisions also affect the optimal level of water $P U_{\text {net }}, W A_{n e t}, O p C H_{n e t}$ and to a lesser extent $O p C H_{n e t}$, that can be achieved through operational optimization of PRVs, FCVs and pump settings for the two seasons. Therefore, because of the magnitude of the search space and the large number of decision variables, the multiple interactions between the objective functions (some complimentary and some conflicting) and heterogeneity of the decision variables, it is not possible to derive a design process that is fully automatic.

In the literature, several methodologies have been proposed to tackle the problem of water network sectorization, often with respect to one decision variable and fewer objectives. For example, motivated to maximize security against contamination events, (Di Nardo et al. 2013) employ graph theory principles and a heuristic optimization to form isolated district meter areas, each of which is supplied by its own source (or sources). Although they use graph theory tools for sectorization, in Scarpa et al. (2016) the two objectives are enhancing the quality of delivered water and reducing the risk of contaminant spread. Most other work also uses graph theory and energy criteria (Di Nardo \& Di Natale 2011; Giustolisi et al., 2015) and some have applied multi-agent systems for finding the best combination of sectors (Herrera et al. 20112, Montalvo et al. 2014) for other multi-objective problems. There are also approaches that try to tackle the problem directly with heuristic optimization algorithms (Di Nardo et al. 2014) and recently with concepts derived from social network theory (Diao et al. 2013). 
A reference to a plethora of other related work on sectorization approaches and specific objectives for sectorization can be found in these references and literature cited therein.

The approach followed in our work is not exclusively based on any one method presented in the literature but a combination of several approaches together with engineering judgement to solve the overall problem in multiple stages. As previously demonstrated in Khedr \& Tolson (2015) for a water distribution system rehabilitation problem, the use of engineering judgement to simplify a rather complex design and operational optimization problem can be very effective. We use graph theory tools to better understand the network topology, visualize the elevation map and identify redundant and isolated network elements, which were corrected before automating some processes. We then optimize iteratively the DMAs using agent swarm optimization. The operational settings of valves and pumps are determined using convex optimization tools. In the following section, we describe the methodologies in detail.

\section{METHODOLOGY}

\section{Engineering principles and Porteau}

The Porteau software solution (2017) is a hydraulic toolkit for Water Distribution Analysis that is developed by IRSTEA research institute in France. It was designed with the help of a ComputerAided Software Engineering tool and object oriented programing. This software is an alternative to the well-known EPANET solution (Rossman, 2000) and it provides different additional tools for analyzing complex WDNs such as simplification of the network, detection of isolated parts and duplicated pipes, and it owns a stochastic module for assessing the hydraulic state at peak period (Piller and Bremond, 2002). The technologic choice and the software possibilities are described in Piller et al. (2011). 
Porteau was first used in the model validation step to identify parts that are isolated from water resources and to check errors in the data file itself. In the original network file provided for the competition, a set of pipes was discovered to be disconnected from the rest of the network. This may cause problems for a hydraulic solver with the presence of customer nodes with no reference of head. One pipe, originally closed (i.e.: Pipe ' 8872 ' in the INP file provided), was opened to avoid problems in hydraulic analysis and force the hydraulic solver to compute a correct rather than arbitrary piezometric head. Another issue we identified was regarding the two tanks 'Tank_3' and 'Tank_4' connected by a short pipe. This caused mass oscillation between the two tanks at each iteration and extended period simulation (EPS) hydraulic equations like in Porteau and EPANET were not able to find a correct physical solution since they ignore pipe inertia; such problems would not be an issue if a rigid water-column model was used (Piller \& Propato, 2006; Nault and Karney, 2016). We also found that this modeling flaw increased considerably the executable time of the EPS. Since the supply flow direction is from 'Tank_3' to 'Tank_4', both in rainy and dry seasons, we placed check valves on the outgoing pipes from both tanks. This prevention of two-way flow in the numerical model prevented the mass oscillation, both decreasing the computational time and giving correct and physically meaningful results. In valve and pump setting optimization problems (see last subsection), the same set of issues were resolved by putting a unidirectional constraint on the flow of the links originating from 'Tank_3' and 'Tank_4'.

Several parallel pipes (with the same positions) were found in the original network data. An attentive check was made to ensure it was not an error coming from the GIS. In Porteau, a connectivity analysis highlights parallel pipes and visualizes them. Then they are manually checked to see if they are different pipes. Since we found no duplicated pipes, the parallel pipes were kept in the model. Five out of service tanks were transformed into junction nodes. Practically, this allows water to cross the valve chamber and bypass the tank. Another abandoned tank ('Tank_7') was isolated because its elevation was too high to be supplied with sufficient pressure coming from another tank or through nodes around it. 
The second step was to analyze the supply routes (Figure 2) and elevation map (Figure 3) to understand the supply network (see Figure 1). In the rainy season, the water has three origins. The source in the south, Cuza WTP, constitutes more than half of the supply and provides water to a large part of the city. The source at the central east of the network, Bachue WTP, is at a low elevation and can only supply the low elevation parts close to it, including Tank_14. Bochica WTP, although it has half the capacity of the south supply, it has the highest elevation and so supplies areas with a large variation in elevation across the north of the network.

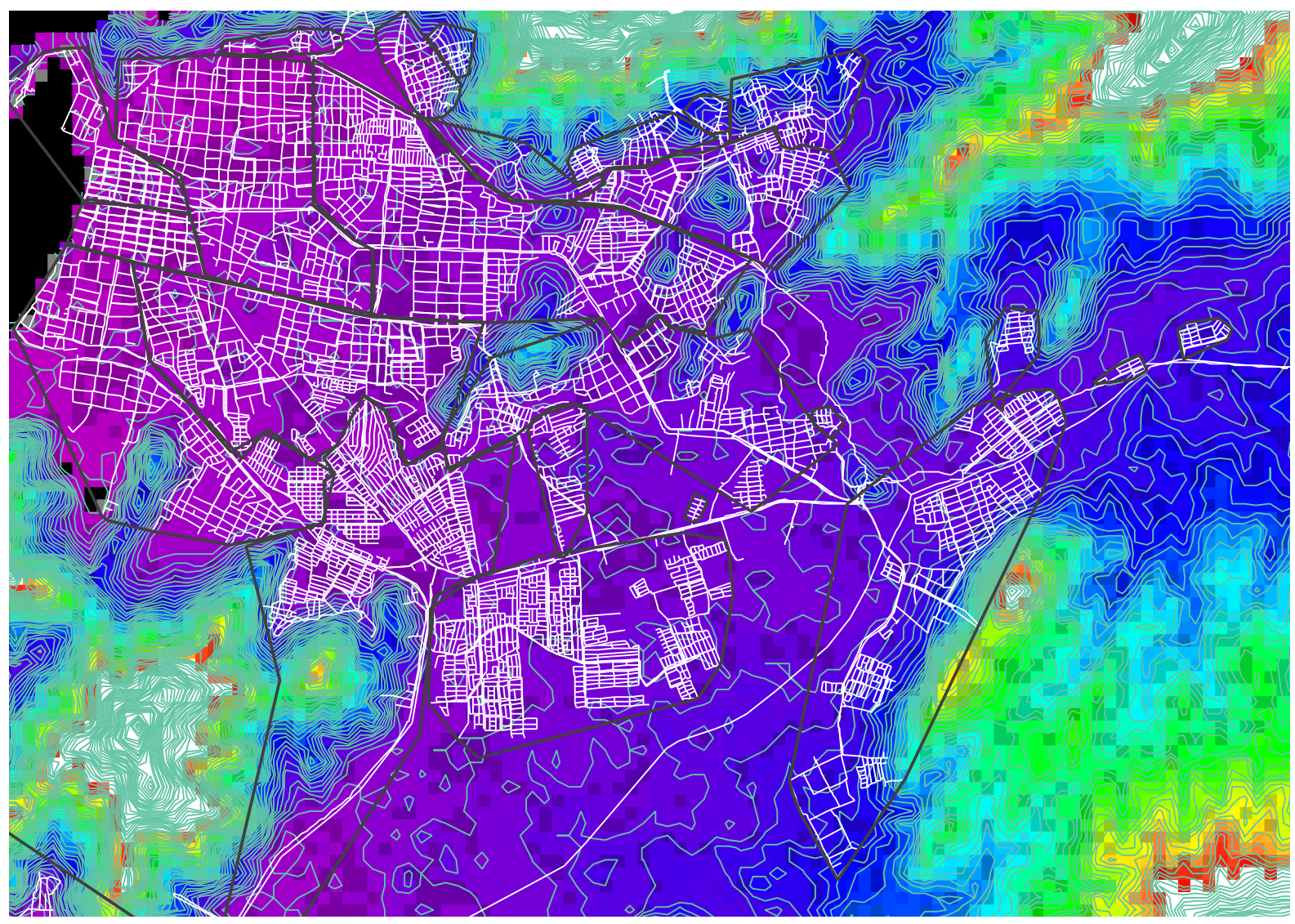

Figure 3:. Altimetry for Downtown E-Town network (network pipes in white; sectors delimited by black lines; elevation contour lines in blue; and the DEM in the background.)

To reduce energy losses in distribution, one of the objectives to be minimized in the new sectorization is pressure uniformity (i.e. the $P U_{\text {net }}$ function in the BWNDMA Problem Description and Rules (BWNDMA, 2016)). This was taken into account in forming a first feasible DMA configuration using engineering know how and Porteau Software for graph sectorization and hydraulic and water quality 
simulation tool. Initially, the elevation information of the network data was used to visualize a digital elevation model (DEM). The contours of homogeneous elevations were then used to form the sector frontiers, where each sector would have a small variance in the elevation. In this way 15 DMAs were chosen as first guess based on elevation similarity, since the other objective specified in the battle was also minimizing the number of DMAs with a minimum number of 15 DMAs. The contours were then moved sufficiently to make sure the DMAs have similar volumes of demand. Closed valves were placed for isolation from the rest of the network, and one main entry point was chosen for each DMA and tagged "meters/compteurs". A graph theoretic tool was used to check that each DMA was connected only through the tagged inlets: using the network incidence matrix of the connected components and the PRIM algorithm (a greedy depth-first-search algorithm) the inlets to each DMA were confirmed (Bartnik \& Minoux, 1986). For double-checking the results, a modified version of the Lee algorithm (Lee, 1961) was used for identifying existing sectors in the network model. The algorithm uses graph decomposition and the information of the network elements for traversing in depth search until the border of a potential sector is reached.

The third step is iterative and includes the use of two different steady state hydraulic models to check the pressure at demand nodes during the peak period. The conventional deterministic models predict too large flow rates in main pipes, which are improbable to occur simultaneously. On the contrary, flow rates in branched pipes supplying few consumers are underestimated by the latter. More representative values can be derived by considering stochastic demands and upper limits of different confidence intervals; we use the stochastic model Opointe in Porteau that exploits peak demand diversity/simultaneity curves (Piller and Brémond, 2002) to more accurately approximate the flow rate at pipes as a function $(a n+b \sqrt{n})$ of the number of domestic consumers, $n$, served. This way a less pessimistic pressure is calculated in the core network that corresponds to a satisfaction risk. 
Additionally, EPANET was used for simulation from within a c-code with conventional analysis of low and peak periods. If the lower constraint limit of $15 \mathrm{~m}$ for pressure was not satisfied it was necessary to adjust the DMA boundary and specify a new DMA with a more homogenous pressure. Following this, another steady state for low demand consumption was run to check for the pressure upper limit of $60 \mathrm{~m}$. Among the main results, it was necessary to reinforce the network with two new pipes of length approximately $800 \mathrm{~m}$ and $1.2 \mathrm{~km}$ (connecting node ' 5675 ' to nodes ' 4466 ' and ' 5374 ', respectively) in order to limit the head loss in supplying the DMA below 'Tank_15'. This high elevation area (e.g., node '4252' has an elevation of $47 \mathrm{~m}$ ) couldn't be supplied with a pressure above $15 \mathrm{~m}$ without violating the $60 \mathrm{~m}$ pressure constraints upstream of it - feasible solutions are obtained only with this reinforcement with new parallel pipes.

The fourth step was to tune the setting of the flow control valves (FCVs) and of the pressure regulating devices (PRVs) that are added at entry points of DMAs. The main role of the FCV is to control the distribution of water and it was possible to set each FCV to the supply of one or several DMAs. This has simplified the problem of finding feasible initial settings for the FCVs. An automatic procedure that requires solving a convex optimization problem was also devised for that task and linked to the pressure uniformity criterion (see the next subsection). This has led to a feasible solution for the rainy season. The average demand of each DMA and the mass balance of each tank were used to determine the initial setting of the FCV.

The solution of the rainy season with pumps working and reduced flows from the water treatment plants was adapted for the dry season. It was necessary to make two kinds of change: closing and opening valves to introduce new water routes (shortcuts) and to supply some tanks with insufficient water inflow with alternative sources. We added a pressure-sustaining valve to raise the pressure in order to limit the water coming from the south source 'Cuza WTP' and force it to go to 'Tank_16' and decreased the flow going to 'Tank_1'. We also reinforced with set pipes of diameter $762 \mathrm{~mm}$ and total length $721 \mathrm{~m}$, starting from 'Fagua_Pump_Station' to transport the supply to 'Tank_12'. 


\section{Sequential Convex Programming for Operational Optimization}

In the problem description and rules for the competition (BWNDMA, 2016), the objectives $D M A_{\text {index }}, D S, C C_{\text {net }}, O p C H_{\text {net }}$, (and implicitly $P U_{\text {net }}$ ) were taken into account only while choosing the DMA configurations in the design iterations as they are not a function of operational setting changes. The operational decisions affect the two objective functions relating to pressure uniformity $\left(P U_{n e t}\right)$ and water age $\left(W A_{n e t}\right)$. By posing the water quality objective function $W A_{n e t}$ as a constraint, i.e. all designs would need to satisfy the stipulated water age limit, this objective function could be removed from the multi-objective design problem. For the dry season, in the operational optimization, we consider the simultaneous tuning of the settings for the FCVs, the PRVs and the flows from the fixed-speed pumps with the two objective functions $P U_{\text {net }}$ and $W A_{\text {net }}$ such that the following (linear constraints) are satisfied:

- pressure constraints of $15 \mathrm{~m}-60 \mathrm{~m}$ for the specified nodes at all time steps,

- flow constraints on all water sources between 0 and the maximum specified, and additional constraint for the two Mohan sources to sum to $206 \mathrm{~L} / \mathrm{s}$,

- tank levels between 10-90\% full, at all time steps, and

- mass balance at all nodes.

In the rainy season, all the above except for the flow from the pumps are considered.

This optimization problem is a difficult nonlinear programming problem (NLP), with non-convex constraints. An optimization method based on the strictly feasible sequential convex programming (SCP) described in (Wright et al., 2015) was used. This SCP method solves the non-convex NLP problem by sequentially solving convex approximations (linearized sub-problems). The convexity of the approximations means each sub-problem can be solved accurately and efficiently. In the optimization problem considered here, we start from feasible PRV and FCV and pump flow settings that were determined in the DMA design stage. Let $x:=\left[\begin{array}{lll}q^{T} & h^{T} & \eta^{T}\end{array}\right]^{T}$ be the vector of the flows in all links $(q)$, the heads at all unknown head nodes $(h)$ and the headloss across the PRVs $(\eta)$, respectively. The NLP for calculating $\eta$ and flows through FCVs at each time instant is as follows: 


$$
\begin{array}{r}
\min _{\eta, q, h} f(h ; \eta, q) \\
\text { subject to: } g(x)=0 \\
\underline{q}_{i}<q_{i} \leq \bar{q}_{i}, \forall i \in N_{F C V} \\
\underline{h}_{j}<h_{j} \leq \bar{h}_{j}, \forall j \in N_{N} \\
\eta_{i} \geq 0, \forall i \in N_{P R V}
\end{array}
$$

where the vector equation $g(x)$ contains the head loss across each link and mass balance equations at all nodes, respectively. Where the link indexed in $g(x)$ is a pump, the head gain across the pump is represented by the pump curve equations (Rossman 2000). The upper and lower bounds on the flows $q_{i}$ represent constraints on flows through FCV from different sources, and the minimum and maximum pressure levels at demand and non-demand nodes and tank level constraints are imposed using the constraints on the heads $h_{j}$. The respective $\eta_{i}$ values are constrained as non-negative for PRVs and positive for pressure sustaining valves (PSVs), where the direction of flow is already determined. For entrance to DMAs with only flow meters, the flow direction constraints can be omitted. At each iteration of the SCP, the nonlinear objective function $f(\cdot)$ and the nonlinear constraints $g(x)$ are approximated by their linearization and the following problem is solved:

$$
\begin{array}{r}
\min _{x} \nabla f\left(x^{k}\right)^{T} x \\
\text { subject to: } \\
\nabla g\left(x^{k}\right)^{T}\left(x-x^{k}\right)+g\left(x^{k}\right)=0, \\
\underline{q}_{i}<q_{i} \leq \bar{q}_{i}, \quad \forall i \in N_{F C V} \\
\underline{h}_{j}<h_{j} \leq \bar{h}_{j}, \forall j \in N_{N} \\
\eta_{i} \geq 0, \forall i \in N_{P R V}
\end{array}
$$

where $x^{k}$ is the variable at the k-th iteration of the SCP, $\nabla f\left(x^{k}\right)$ and $\nabla g\left(x^{k}\right)$ are the gradients (i.e. partial derivatives) of the objective function and constraints, respectively, computed at current iterate $x^{k}$ and $x$ is the solution we seek, at which we shall compute the linearized gradients at the next iterate $k+1$. In addition to these, we impose flow direction constraints for the supply pipes (i.e., the FCVs from the sources), and inlet PRVs by setting the corresponding lower bound $\underline{q}_{\mathrm{i}}$ to zero. 
In the optimization problem (1), the flows from the pumps at Fagua and Mohan water treatment plants and the head difference across the pumps are decision variables, constrained by the corresponding pump curves. In the SCP subproblems, the heads are approximated using the linearization of the pump curve at a given flow. In Figure 4, we show different approaches that can be used to approximate the pump curves. A simple approximation of the pump-curve (left side of Figure 4a and Figure 4b) can be derived by drawing a line between the head and flow axis intercepts; this underestimates the head of the pump for a given flow and sometimes can result in violation of the maximum head constraints. Alternatively, (right side of Figure $4 \mathrm{a}$ and Figure $4 \mathrm{~b}$ ), a least-squares fit of the pump curve can overestimate or underestimate the head depending on the flow rate. A first-order Taylor linearization of the pump-curve around flow $q^{k}$ (at the k-th iteration of the SCP) gives an overestimator for the head that is tight near $q^{k}$. From simulations, we found that the last linearization had the least hydraulic infeasibility, a faster convergence for the SCP and therefore it was adopted; the details of this pump approximation approach and how the tank levels are discretized using a first-order finite-difference model is described in (Menke et al., 2016).

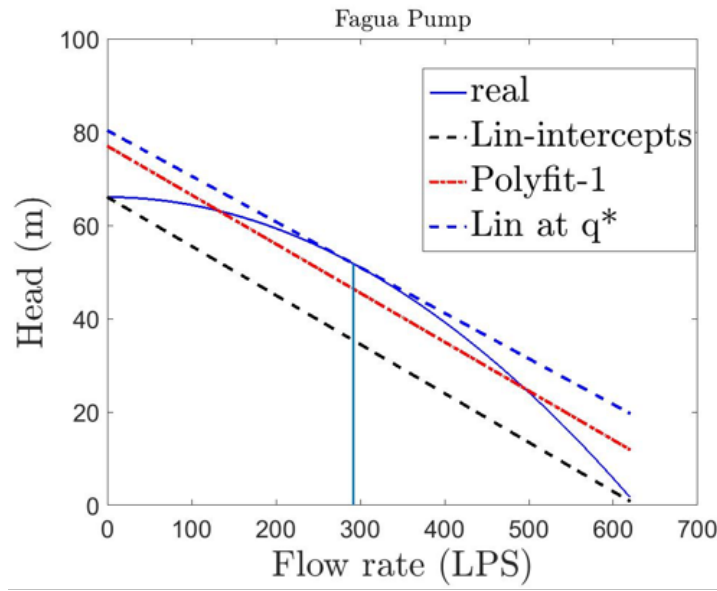

(a)

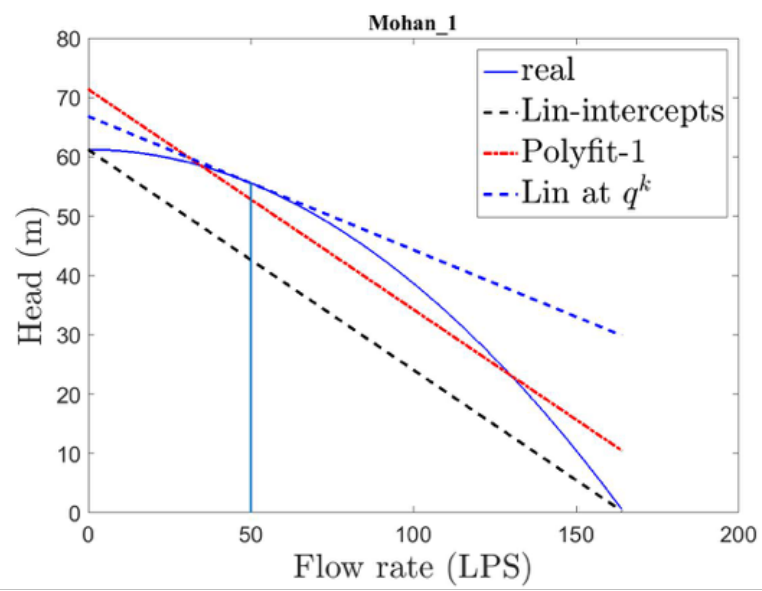

(b)

Figure 4: (a) Different linear approximations for the Pump Curve of Fagua compared to the given quadratic pump curve labelled 'real'. (b) Different linear approximations for the Pump Curves of Mohan compared to the given quadratic pump curve labelled 'real'. The vertical line shows the flow $\mathrm{q}^{*}$ at the current iterate.

Three more aspects of the SCP application in our implementation that are important to mention are: 
- The objective function $W A_{n e t}$ was ignored for the SCP because it was mostly zero when perturbing the feasible initial solution from the design subproblem. Moreover, ignoring this objective function means that the water quality simulations are not needed at each iteration of the SCP but only an extended period hydraulic simulation to check feasibility in taking a step in decision variables. This results in faster CPU time of the SCP. $W A_{n e t}$ was checked once the SCP had converged.

- The PRV technology used by the utility are specified to have fixed-head settings that change only once from dry to rainy season and vice versa. Therefore, in the operational optimization we enforce a constraint on the pressure at the downstream nodes of the regulating PRVs to be fixed in time. Most times, this caused infeasibility in the linearized subproblems of the SCP, even when starting from a feasible point. The linearized feasibility spaces at different times (and therefore at different states for flows and pressures) seem to no longer have an intersection with a fixed head constraint for the PRVs. This was relaxed by putting a penalty term for temporal changes in the downstream heads of PRVs instead. However, it is not clear how to find appropriate penalty weights to guarantee that we don't introduce too much suboptimality in the objective $P U_{\text {net }}$. As a result, the SCP's results do not always give significant improvement. It is also not clear how optimal our initial feasible guess was since we are using a local optimization solver. Therefore, this is left as an interesting study for future work.

- The nonlinear objective $P U_{\text {net }}$ was studied by decomposing it into the two parts:

$$
\sum_{j=1}^{M}\left[\frac{1}{N} \sum_{i=1}^{N} \frac{\left(P_{i, j}-P_{\min }\right)}{P_{\min }}+\frac{\sqrt{\frac{\sum_{i=1}^{N}\left(P_{i, j}-P_{A v j}\right)^{2}}{N}}}{P_{A v j}}\right]
$$

Where $P_{\min }$ was fixed to be $15 \mathrm{mH} 2 \mathrm{O}, P_{i, j}$ is the pressure at node $i$ and time $j$; and $P_{A v j}$ is the average pressure at time $j$ for all the nodes. 
Using the semidefiniteness of the numerator in the second term of equation (3) and the positivity of its denominator, perhaps it is possible to show convexity of $P U_{n e t}$, which simulations seem to imply. Nonetheless, we use a linearization of $P U_{n e t}$ in the SCP.

\section{Network design optimization by an ASO method}

Creating sectors in a water network is a challenging problem from the engineering point of view. Even more so when it is approached using optimization methods trying to find optimal DMA configurations:

- Classic optimization based on derivatives would be very limited for solving this problem as they need rigorous formulation and the decision variable should be continuous.

- Checking all alternatives by enumerating solutions is infeasible in terms of calculation time as the problem scales combinatorically (Pecci et al., 2016)

- The use of "pure" evolutionary algorithms would have difficulties to find good solutions (in a reasonable amount of time/resources) for a "large size" problem like this one, because for example GA is sensitive to the initial population used and Genetic algorithms do not scale well with complexity.

- Hybrid evolutionary approaches that incorporate engineering experience and other more deterministic methods for solving sub-problems are considered as an option.

Agent Swarm Optimization (ASO) (Montalvo et al., 2010) introduced a new agent that reproduces the behavior of the Louvain method for community detection (Blondel et al., 2008). The Louvain method maximizes a modularity index resulting in the best possible grouping of nodes:

$$
\mathrm{Q}=\frac{1}{2 m} \sum_{i j}\left[A_{i j}-\frac{k_{i} k_{j}}{2 m}\right] \delta\left(c_{i}, c_{j}\right), \text { where }
$$

- $A_{i j}$ represents the edge weight between nodes i and j,

- $\quad k_{i}$ and $k_{j}$ are the sum of the weights of the edges attached to nodes $\mathrm{i}$ and $\mathrm{j}$, respectively,

- $m$ is half the sum of all edge weights in the graph,

- $c_{i}$ and $c_{j}$ are the communities of the nodes $\mathrm{i}$ and $\mathrm{j}$ respectively,

- $\delta$ is a Dirac delta function that gives 1 if $\mathrm{ci}==\mathrm{cj}$, else zero. 
Classic applications related to social network can use, for example, the number of messages between community members for weighting edges between nodes. In the case of water network sectors, this research has been using the water demand at nodes as a first weighting factor. For joining smaller communities when the modularity index was not able to be increased anymore the $\mathrm{Z}$ coordinate (or elevation) was used as weighting factor. These decisions had the intention to find sectors with similar demand and with nodes located at similar altitude. The action of the agent reproducing the Louvain method was combined with other type of agent reproducing the behavior of a modified Lee algorithm (Lee, 1961) for finding the limits of potential sectors and deciding where to put PRV valves. This behavior was also used in this research for checking solutions created just based on engineering experience. ASO calculation and network visualization were done using Water-Ing, software from Ingeniousware $\mathrm{GmbH}$. The software is open to extensions and available for free in a community edition (https://ingeniousware.com/software/about?name=watering).

The settings of the PRVs placed at DMA boundaries for separating sectors are not included directly in the process of creating sectors. Nevertheless, the results of the objective function after the PRV settings are defined is sent back to the ASO as reference in order to improve the sectors created. During the time of this research, ASO was partially used to improve the sectorization solution as because of limited time, it was not possible to automate the whole process of creating sectors taking into account all the details of the objective functions. Nevertheless, the agents included in ASO helped on the evaluation/verification of the ideas emerging from engineering experience that were used to solve the problem.

\section{SUMMARY and DISCUSSION OF RESULTS}

In Figure 5, we show DMA configurations of the main North part of the network where the colors were chosen from a library by the Porteau software to distinguish each DMA. It can be observed that there is a large variation of elevation within small distances in the North. As a result, it was necessary to sectorize the areas into smaller DMAs so that the pressure can be controlled within the given limit of 
(15m-60m pressure) using PRVs, each DMA acting like an "irrigation terrace" used in agriculture as we go uphill. Although one of the objectives by the battle organizers was to have the smallest number of DMAs possible (but above 15 in number), this also explains the relatively large number of DMAs in this area, which has resulted in some DMAs having relatively smaller average volume demand compared to bigger DMAs with higher volume of consumption. Therefore, the demand similarity objective $D S$ was increased to maximum to make sure the conflicting pressure similarity objective is reduced, and pressure constraints were satisfied.

For the south part (see Figure 6) and in our sectorization solution, the water is coming from Tank 15 (depicted with the dark-blue square in the center bottom). It is important to highlight the necessary reinforcement/reorganization is the most important intervention we have done in term of implementation costs. It consists of pipe reinforcement with roughly $2 \mathrm{~km}$ of pipes (top thick green line) to limit the head loss at the lower elevation and satisfy pressure constraints at the high altitude in the ends of DMA 4. Because of the large variation in elevation in in this area, we created three DMAs to sectorize this area into three areas of more homogeneous elevation. 


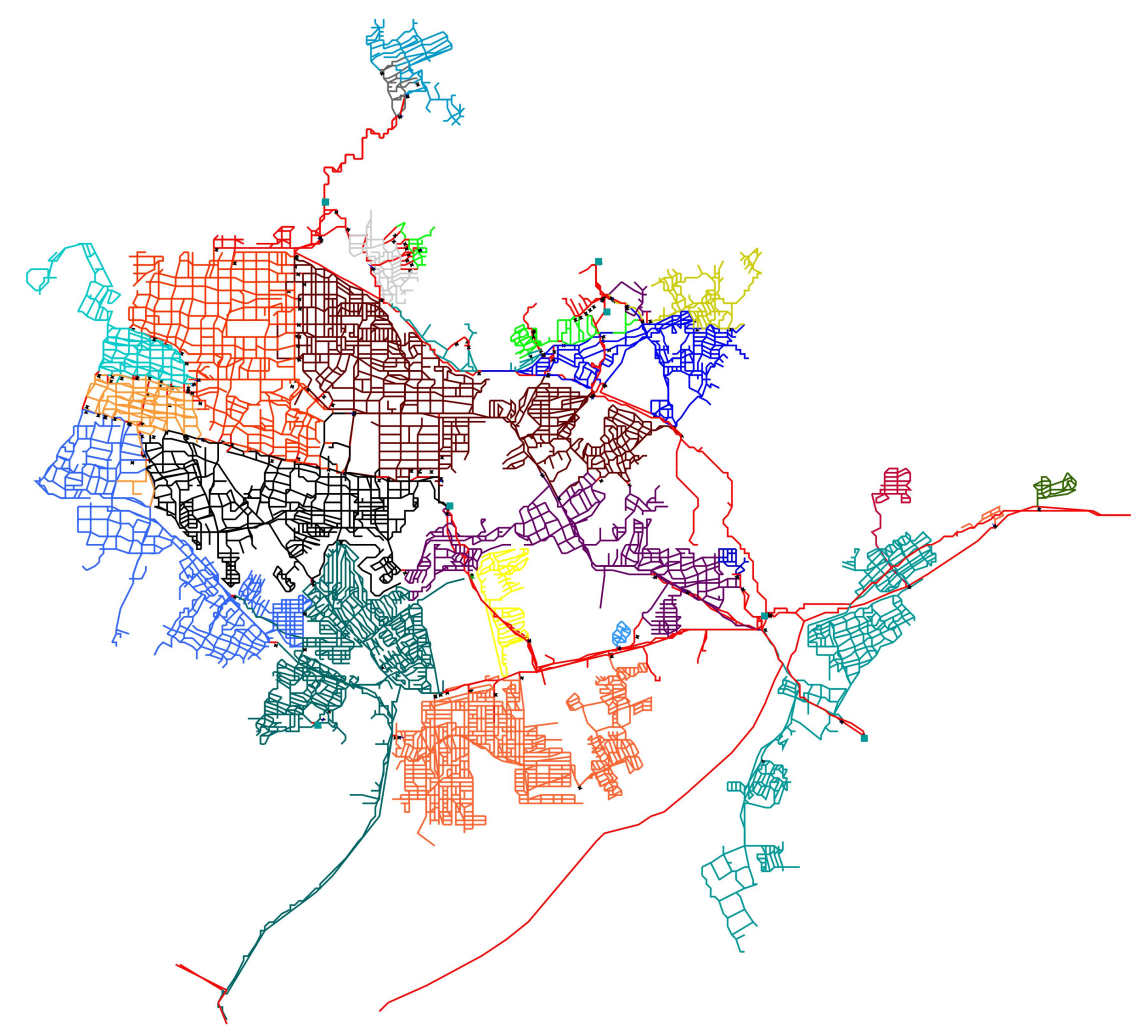

Figure 5: Final DMA splitting result for the main North part of E-Town network

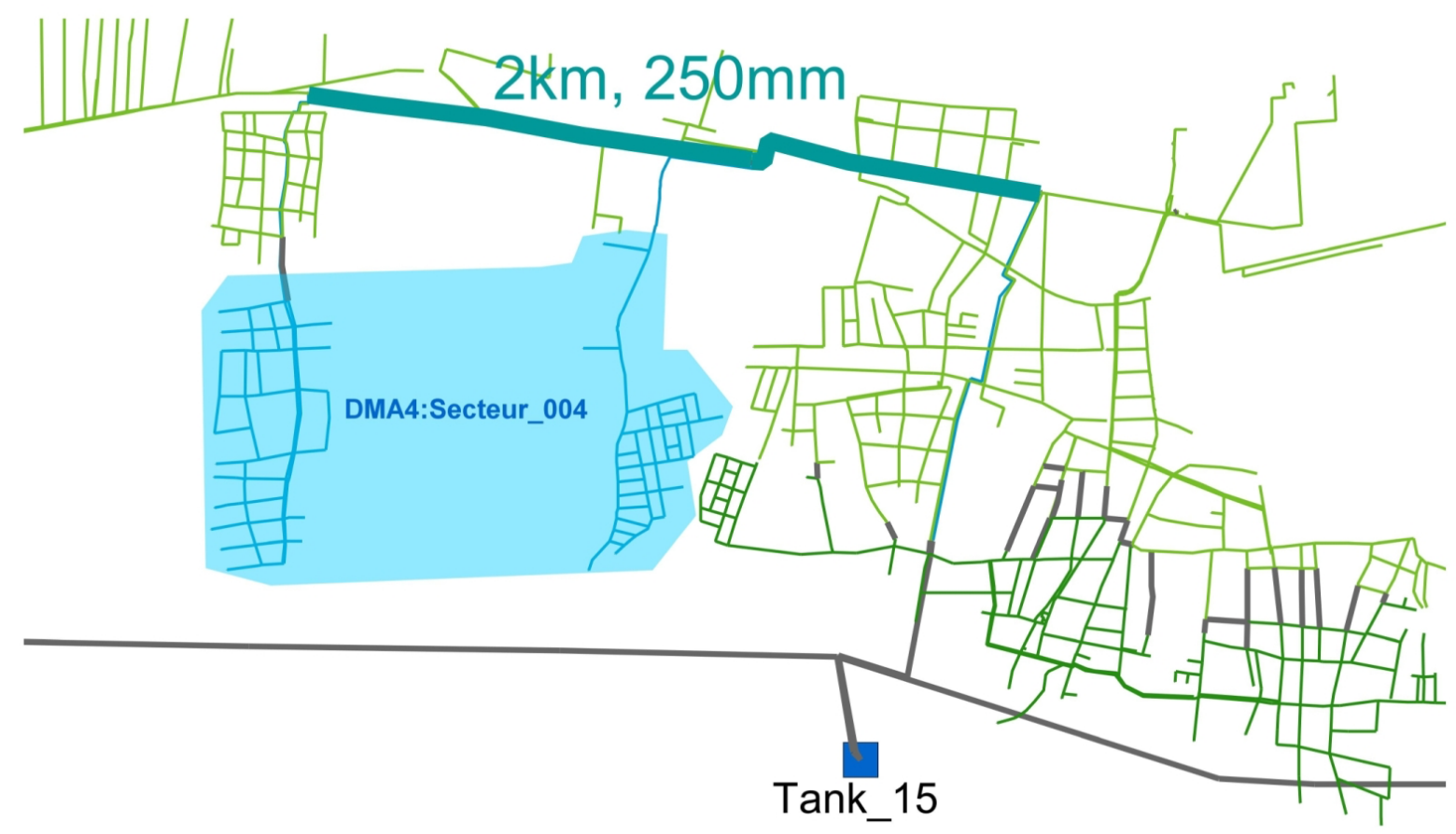

Figure 6: Final DMA 4 for the South part of E-Town network

We show in Figure (7) the center part of the network, where in the rainy season, the water comes mainly from Bachue (the very right of Figure 7). In the dry season, this supply is halved in capacity and so was complemented by Mohan pumping station. In this competition, the pumping stations are specified to 
contain only fixed-speed pumps. To increase redundancy and flexibility in supply, we recommend the pumps (to have higher head specifications) and introduce variable speed pumps, which can be operated more efficiently with resultant savings of energy (Wu et al. 2011). This can be exploited even further if the water resources (aquifers) at Mohan have capacity to supply more water.

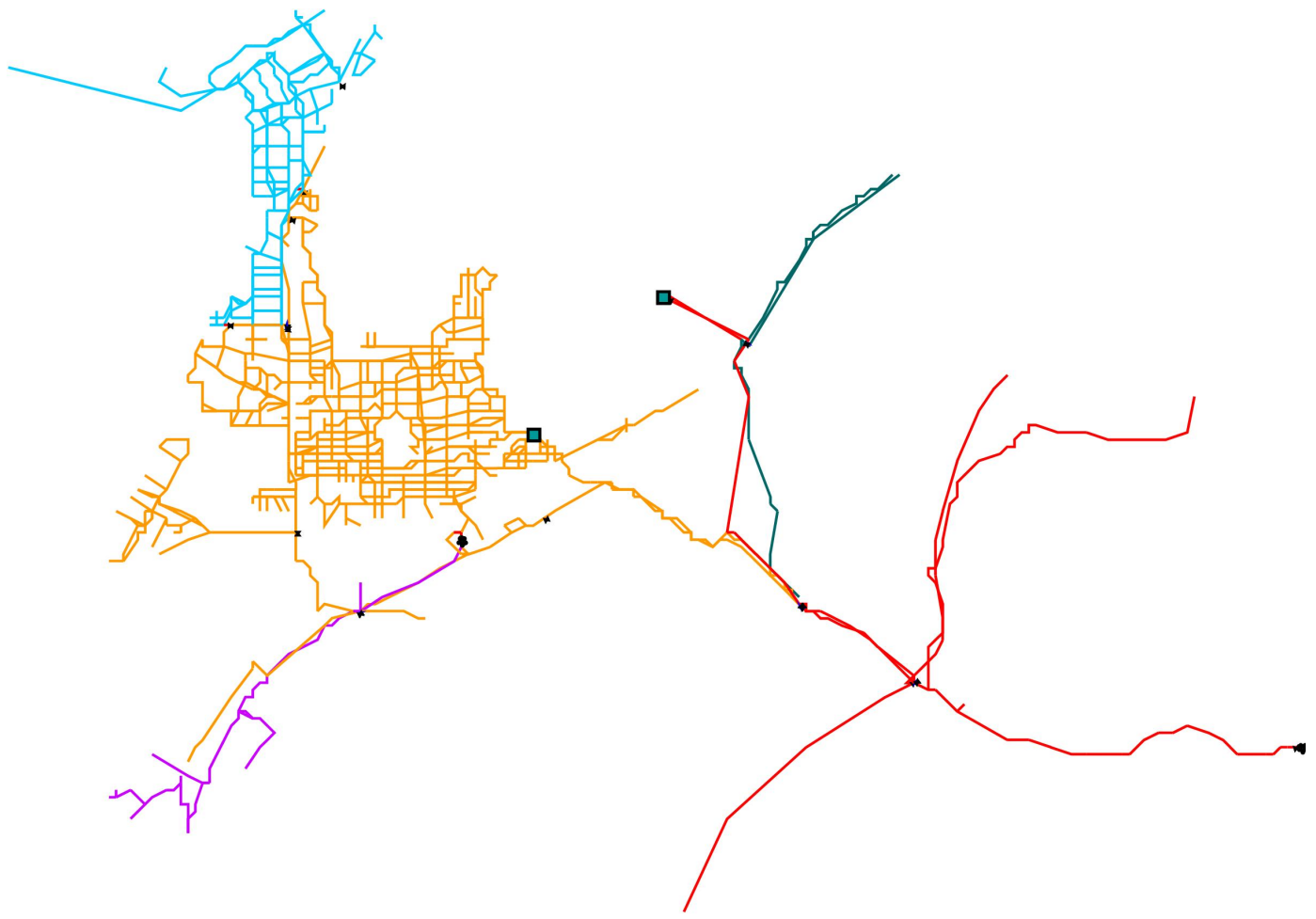

Figure 7: Final DMA splitting for the central part of E-Town network

In summary, the following were also achieved in the final configuration and optimized operational settings:

- In Table 1, we show the decomposition of $P U_{n e t}$ into the first and second elements of the sum in Equation (3), labelled PUnet_Pmin PUnet_Pav, respectively. We note that some DMAs have high deviation from the minimum pressure because they are either in a sector of low elevation near the sea ('Secteur_005', 'Secteur_010' in NW) or possess high variation in elevation and are close to a source supplying other DMAs ('Secteur_012' and 'Secteur_020' at the entrance of the Northern part, see also Figure 8). Note also that 'Secteur_012' is close to 'Tank_16' with nodal pressures close to the $60 \mathrm{~m}$ upper limit at times). 


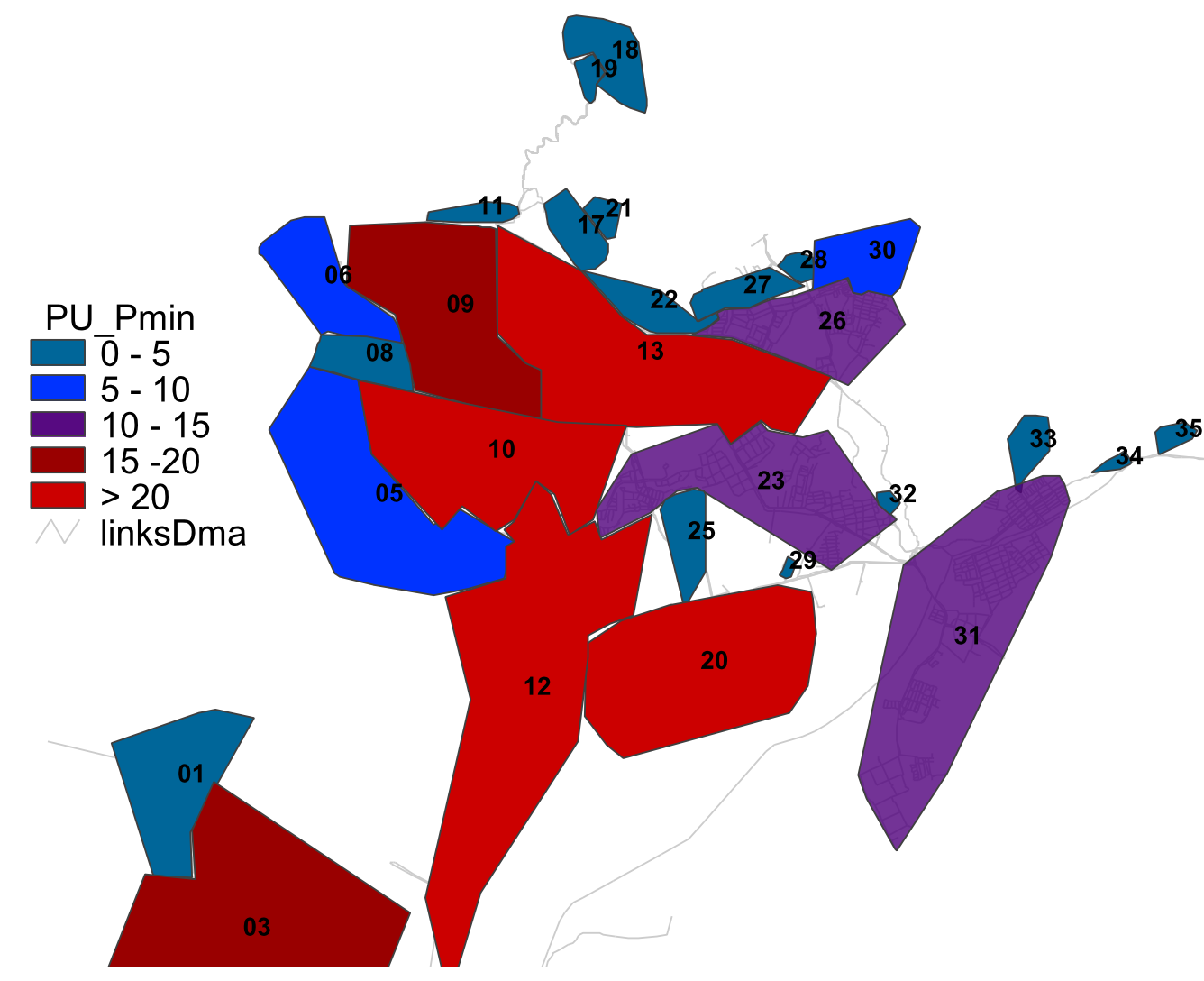

Figure 8: Mean pressure deviation from PUnet_Pmin for the North part of E-Town network

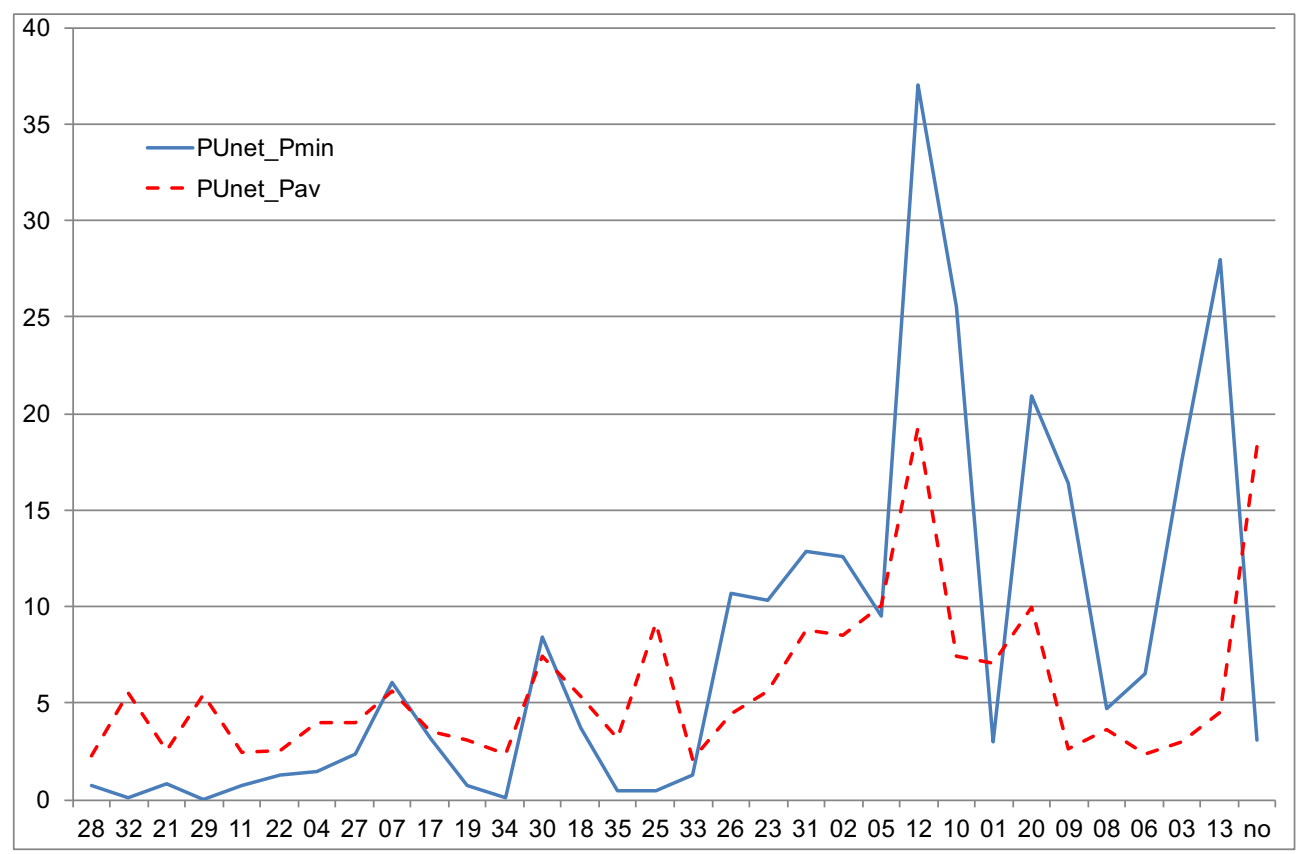

Figure 9: The two PU contributions for all DMAs contributions of E-Town network; the DMAs are sorted by total demand. 
- The water age objective $\left(W A_{n e t}\right)$ is close to zero $(0.005$ hours $)$ in both seasons, as there are only 20 violations of the 60 -hour maximum limit.

- By making use of a graph simplification tool and engineering judgement, it was possible to identify the main flows and bottlenecks. This has allowed changing the network with minimal pipe interventions (11 in total but with only 5 with significant lengths). Similarly, the operational changes from one season to another have been kept to only 14 (with five main pipes status changes, eight FCV setting changes and three PRV setting changes). The details of all these are summarised in the Excel files submitted as supplementary material together with the optimized network models in EPANET INP format.

- Similarly, we have tried to minimize the capital cost of intervention with valves (see Table 2). Although we use 52 valves in the network, 13 of the PRVs located at entry point of the DMAs are completely open and not doing any regulation. They only represent water meters at their respective DMA entry point, where we are required to measure the volume of water going through. EPANET does not offer the possibility to include water meters and that's why they are represented in our solution as totally open PRV without any regulation.

\section{SUMMARY AND PERSPECTIVES}

The objective of this paper is to share our experience with the civil engineering and academic community in facing the challenging Battle of Water Networks District Meter Areas problem. We have adopted three main strategies:

The first one consists of using our experience and skills in network modeling as much as possible. For that, it was very important to understand how the supply of tanks and demand nodes may work in rainy and dry seasons. The steps taken included:

- model validation, 
- supply route and altimetry analysis,

- use of a deterministic and stochastic hydraulic model to test the pressure constraints for each candidate DMA configuration,

- tuning the setting of the FCVs and of the PRVs.

This strategy has allowed the derivation of a feasible solution with regards to the pressure and tank level-range constraints. It was the most time consuming and has required inventiveness. Our experience was also used to simplify the problem in terms of the number of variables and objectives and to help the ASO with appropriate rules.

The second strategy was to use a metaheuristic agent-swarm method to explore other solutions that are not intuitive and so were not exploited in engineering judgment. The method moves some elementary pieces of network and analysis the different combinations. A single hydraulic steady state was run to check the pressure constraint feasibility and water age constraints. No substantial improvement was achieved due to the lack of automation of the overall sectorization process with ASO.

The third and last strategy was to solve the operational optimization problem for the setting of PRVs and FCVs, and for the pump working point by sequential convex programming method. Further studies are needed to guarantee significant decreases in the objective or use relaxation approaches for global optimality studies (Pecci et al. 2017).

The final solution consists of a reinforcement with $3 \mathrm{~km}$ of pipes at a cost of $141 \mathrm{k} \$$. In sectorizing the network, we placed three kinds of control and metering devices: PRVs that are regulating the DMAs are placed at the sector entrances, metering devices that we represent by open PRVs, and open PRVs at the outlets for metering what is leaving the DMAs. The valves cost $208 \mathrm{k} \$$ in total. There is no tank cost since we do not recommend new tanks or increasing the capacity of existing ones; the total cost is only approximately $349 \mathrm{k} \$$. The water age at every node is lower than 60 hours so that our solution is 
optimal for this objective, with only insignificant violations of 0.05 hours aggregated over all nodes over seven days. Satisfying the objectives to the average pressure and increasing the pressure uniformity in each and across the DMAs has led to the creation of additional smaller sized DMAs compared to the solution derived at the first stage. Finally, we have 31 DMAs in total.

Future research will study further the use of ASO and deterministic optimization tools, as well as their efficient coupling to further explore the space of possibilities. We have also provided as supplementary material to this paper, the INP files of our results and a summary of the results in Excel files containing the scores of the objective functions set, the definition of the sectors, nodal pressures, the tank level at each time-step, and all these for the two seasons. Visual Basic and C codes for running EPANET and generating the analysis on the networks are available on request.

It should be noted that, although the algorithms and software tools used have were vital in solving a large-scale problem that is too complex to tackle manually, they were complimentary to sound engineering judgement rather than completely substituting it. An essential core of the solutions was also the result of applying experience and engineering knowledge. 


\section{REFERENCES}

Bartnik, G., \& Minoux, M. (1986). Graphes, algorithmes, logiciels. Bordas.

Deuerlein, J., Elhay, S., and Simpson, A. (2015). "Fast Graph Matrix Partitioning Algorithm for Solving the Water Distribution System Equations." Journal of Water Resources Planning and Management, 0(0), 04015037.

C. Y. Lee (1961). An Algorithm for Path Connections and Its Applications. In: IRE Transactions on Electronic Computers. EC-10, Nr. 2, 1961, S. 346-365.

Di Nardo, A., Di Natale, M., Santonastaso, G. F., Tzatchkov, V. G., \& Alcocer-Yamanaka, V. H. (2013). Water network sectorization based on graph theory and energy performance indices. Journal of Water Resources Planning and Management, 140(5), 620-629.

Di Nardo, A. \& Di Natale, M. 2011 A heuristic design support methodology based on graph theory for district metering of water supply networks. Engineering Optimization 43 (2), 193-221.

Diao, K., Zhou, Y. \& Rauch, W. 2013 Automated creation of district metered area boundaries in water distribution systems. J. Water Res. Pl-ASCE 139 (2), 184-190.

Giustolisi, O., Berardi, L., Laucelli, D., Savic, D., \& Kapelan, Z. (2015). Operational and tactical management of water and energy resources in pressurized systems: Competition at WDSA 2014. Journal of Water Resources Planning and Management, 142(5), C4015002.

Herrera, M., Izquierdo, J., Pérez-García,R. \& Montalvo, I. 2012 Multiagent adaptive boosting on semisupervised water supply clusters. Environ. Modell. Softw. 50 (August 2012), 131-136.

Khedr, A., \& Tolson, B. (2015). Comparing optimization techniques with an engineering judgment approach to WDN design. Journal of Water Resources Planning and Management, 142(5), C4015014. Menke, R., Abraham, E., Parpas, P., \& Stoianov, I. (2016). Demonstrating demand response from water distribution system through pump scheduling. Applied Energy, 170, 377-387.

Montalvo, I., Izquierdo, J., Campbell, E., Pérez-García, R. (2014) “Cloud-based Decision Making in Water Distribution Systems" Procedia Engineering, 89, 488-494 
Nault, J., and Karney, B. (2016). "Improved Rigid Water Column Formulation for Simulating Slow Transients and Controlled Operations." Journal of Hydraulic Engineering, 04016025.

BWNDMA Committee. (2016, May). "Battle of Water Networks DMAs (BWNDMA), Problem Description and Rules", Water Distribution Systems Analysis Conference 2016, Cartagena de Indias, Colombia, July 24 - 28, 2016. Retrieved from https://wdsa2016.uniandes.edu.co/index.php/battle-ofwater-networks.

Pecci F, Abraham E, Stoianov I (2015). Scalable Pareto set generation for multiobjective co-design problems in water distribution networks: a continuous relaxation approach, Structural and Multidisciplinary Optimization, In Press, 2015, doi: 10.1007/s00158-016-1537-8.

Pecci F, Abraham E, Stoianov I (2017). Outer approximation methods for the solution of co-design optimisation problems in water distribution networks, IFAC, 2017.

Piller, O., \& Propato, M. (2006, August). Slow transient pressure driven modeling in water distribution networks. In Proc. Water Distribution Systems Analysis Symposium (pp. 1-13).

Piller, O., and Brémond, B. (2002). "A Stochastic Model for Peak Period Analysis of Pipe Networks." ASCE Environmental \& Water Resources Systems Analysis (EWRSA), Roanoke (VA), US.

Piller, O., Gilbert, D., Haddane, K., and Sabatié, S. (2011). "Porteau: An Object-Oriented Programming Hydraulic Toolkit for Water Distribution System Analysis." Urban Water Management: Challenges and Opportunities. Savic Dragan, Kapelan Zoran and Butler David (Eds), Exeter, UK, printed by Centre for Water Systems, University of Exeter, Volume 1/3, 27-32.

Porteau, Irstea (2017), “http://porteau.irstea.fr/”, in French accessed on February 18, 2017.

Rossman, L. A. (2000): “EPANET 2 Users manual.”, EPA/600/R-00/057, Environmental Protection Agency, Cincinnati, Ohio, USA.

Scarpa, F., Lobba, A., \& Becciu, G. (2016). Elementary DMA Design of Looped Water Distribution Networks with Multiple Sources. Journal of Water Resources Planning and Management, 142(6), 04016011. 
Wright, R., E. Abraham, P. Parpas, and I. Stoianov (2015), Control of water distribution networks with dynamic DMA topology using strictly feasible sequential convex programming, Water Resources Research, 51, 9925-9941.

Wu, W., Simpson, A. R., Maier, H. R., \& Marchi, A. (2011). Incorporation of variable-speed pumping in multiobjective genetic algorithm optimization of the design of water transmission systems. Journal of Water Resources Planning and Management, 138(5), 543-552.

\section{FIGURE CAPTIONS}

Figure 1: E-Town network Map with resources and used tanks.

Figure 2: The original supply routes for the E-Town network (BWNDMA 2016)

Figure 3:. Altimetry for Downtown E-Town network (network pipes in white; sectors delimited by black lines; elevation contour lines in blue; and the DEM in the background.

Figure 4: (a) Different linear approximations for the Pump Curve of Fagua compared to the given quadratic pump curve labelled 'real'. (b) Different linear approximations for the Pump Curves of Mohan compared to the given quadratic pump curve labelled 'real'. The vertical line shows the flow $\mathrm{q}^{*}$ at the current iterate.

Figure 5: Final DMA splitting result for the main North part of E-Town network

Figure 6: Final DMA 4 for the South part of E-Town network

Figure 7: Final DMA splitting for the central part of E-Town network

Figure 8: Mean pressure deviation from Pmin for the North part of E-Town network

Figure 9: The two PU contributions for all DMAs of E-Town network; the DMAs are sorted by total demand.

\section{TABLES}


Table 1. Comparison of PUnet objective function elements for all the 31 DMAs.

\begin{tabular}{|c|c|c|c|c|c|}
\hline DMA & $\begin{array}{l}\text { demand } \\
\text { m3/7 days }\end{array}$ & nbNode & nbDemandNode & PUnet_Pmin & PUnet_Pav \\
\hline Secteur_028 & 1549.31 & 25 & 25 & 0.6987 & 2.2601 \\
\hline Secteur 032 & 1759.63 & 29 & 29 & 0.0691 & 5.4905 \\
\hline Secteur_021 & 2006.85 & 31 & 26 & 0.8269 & 2.5155 \\
\hline Secteur_029 & 2301.72 & 26 & 26 & 0.0235 & 5.4707 \\
\hline Secteur_011 & 4908.42 & 44 & 37 & 0.7508 & 2.4374 \\
\hline Secteur_022 & 5191.81 & 48 & 36 & 1.3208 & 2.5766 \\
\hline Secteur_004 & 5383.49 & 118 & 62 & 1.4741 & 4.0094 \\
\hline Secteur_027 & 7897.8 & 88 & 74 & 2.3604 & 4.0048 \\
\hline Secteur_007 & 9387.84 & 193 & 162 & 6.1162 & 5.6021 \\
\hline Secteur_017 & 9578.36 & 100 & 94 & 3.2133 & 3.5324 \\
\hline Secteur_019 & 10279.01 & 50 & 39 & 0.7096 & 3.1027 \\
\hline Secteur_034 & 10739.73 & 14 & 12 & 0.1432 & 2.3412 \\
\hline Secteur_030 & 11089.48 & 246 & 224 & 8.4334 & 7.414 \\
\hline Secteur_018 & 12335.58 & 145 & 115 & 3.7259 & 5.3124 \\
\hline Secteur_035 & 14782.33 & 34 & 30 & 0.4497 & 3.1806 \\
\hline Secteur_025 & 15004.06 & 103 & 97 & 0.4734 & 9.1526 \\
\hline Secteur_033 & 15567.98 & 38 & 34 & 1.2717 & 2.1086 \\
\hline Secteur 026 & 23114.2 & 448 & 377 & 10.6525 & 4.4648 \\
\hline Secteur_023 & 54159.39 & 514 & 440 & 10.3694 & 5.6524 \\
\hline Secteur_031 & 54601.91 & 498 & 410 & 12.8299 & 8.7903 \\
\hline Secteur_002 & 57795.21 & 557 & 409 & 12.5499 & 8.492 \\
\hline Secteur_005 & 71648.25 & 572 & 507 & 9.5529 & 10.0936 \\
\hline Secteur_012 & 73703.9 & 934 & 773 & 37.0166 & 19.2645 \\
\hline Secteur_010 & 77886.19 & 852 & 713 & 25.5174 & 7.4012 \\
\hline Secteur_001 & 81519.5 & 249 & 186 & 3.0262 & 7.093 \\
\hline Secteur_020 & 83853.16 & 1055 & 898 & 20.8845 & 9.9377 \\
\hline Secteur_009 & 84905.37 & 606 & 501 & 16.4216 & 2.6549 \\
\hline Secteur_008 & 85158.52 & 250 & 198 & 4.678 & 3.6356 \\
\hline Secteur_006 & 87365.95 & 338 & 217 & 6.5152 & 2.3366 \\
\hline Secteur_003 & 88830.7 & 708 & 567 & 17.5792 & 3.0187 \\
\hline Secteur_013 & 90513.91 & 1045 & 891 & 27.9382 & 4.5598 \\
\hline noDma & 9112.37 & 1164 & 41 & 3.0443 & 18.2869 \\
\hline
\end{tabular}

Table 2. Intervention types and associated cost.

\begin{tabular}{|l|l|}
\hline Intervention type & Implementation cost in \$ \\
\hline Pipes & 141188 \\
\hline Tanks & 0 \\
\hline Valves & 208142 \\
\hline Total & $\mathbf{3 4 9 3 3 0}$ \\
\hline
\end{tabular}

